\section{Cutting the synaptic cord}

\section{By Lev Osherovich, Senior Writer}

The earliest steps in Alzheimer's disease have proven tricky to target with therapeutics because they occur as part of normal brain activity and before symptom onset. This pathway now has been fleshed out by a trio of papers that have mapped a sequence of synaptic events leading to $\beta$-amyloid production and its sequelae, pointing to new therapeutic targets and drug delivery strategies (see Figure 1, "Mapping early Alzheimer's disease events and targets").

Two studies ${ }^{1,2}$ address the endosomal processing events that occur within neuronal cells to convert amyloid precursor protein (APP) to $\beta$-amyloid $(A \beta)$, which is the major constituent of the amyloid plaques that are the hallmarks of $\mathrm{AD} .^{3}$

The third study tackles the consequences of $A \beta$ exposure on signaling pathways in neurons and identifies a phosphoinositide called phophatidylinositol-4,5-bisphosphate $\left(\mathrm{PIP}_{2}\right)$ as a potentially neuroprotective target. ${ }^{4}$ The work has led to the formation of drug discovery company Smart Biosciences Inc.

\section{Getting in}

The first report, published in Neuron by a group at Washington University School of Medicine, shows how synaptic activity increases the production of $A \beta$ fragments. The study used an in vivo microdialysis procedure to monitor levels of $A \beta$ in the fluid that surrounds brain cells. The researchers discovered that electrical stimulation of neurons caused a sharp spike in the production of $A \beta$ found in the fluid surrounding brain cells.

Lead author John Cirrito, research instructor at Washington University's Department of Neurology, told SciBX that his work shows in real time how the toxic $A \beta$ fragment is made. The key step, Cirrito said, is endocytosis of membrane-bound APP upon neuron stimulation.

The proof was the finding that blocking endocytosis with an inhibitor of the protein dynamin halted the production of $A \beta$ in vivo. Dynamin is required for endocytosis in neurons and other cell types.

In endocytosis, regions of the plasma membrane are encapsulated by a complex of proteins that pull a portion of the membrane into the cell to form an endosome. Endocytosis is a normal step in synaptic transmission, but problems arise when membrane-bound, diseaseassociated proteins such as APP become incorrectly involved.

"This work shows in a living animal that endocytosis is really necessary for making A $\beta$," Cirrito told SciBX.
Gilbert Di Paolo, assistant professor of pathology at Columbia University Medical Center, said the Cirrito paper answers "the fundamental question of where $A \beta$ fragments are generated." Di Paolo called the study an "interesting proof of concept" that shows that targeting endocytosis could potentially be a therapeutic strategy for treating $\mathrm{AD}$.

The next step is to figure out how to selectively decrease endocytosis without interfering with normal synaptic transmission. This is not out of the question, as neurons treated with dynamin inhibitors still could function despite the blockade of endocytosis.

"Inhibiting dynamin did not mess up synaptic transmission," said Cirrito. "This was a surprise."

Cirrito speculated the dynamin inhibitor only partially blocked endocytosis while still greatly decreasing the amount of unwanted APP internalization.

Although dynamin inhibitors showed good therapeutic properties in neurons, the protein may not be an ideal therapeutic target because of its essential role in endocytosis in other cell types.

"Dynamin is involved in so many endocytic processes that it would be difficult to target it," Di Paolo told SciBX. "But there are probably some endocytic factors that would be more specifically involved" in APP internalization that would make better targets.

Cirrito thinks one such target may be an APP endocytosis cofactor called low density lipoprotein-related protein 1 (LRP1). LRP1 recruits cholesterol-bearing apolipoprotein to the plasma membrane of neurons with the help of APP. ${ }^{5}$ Cirrito told SciBX that experiments to test the role of LRP1 in A $\beta$ production are underway.

Cirrito's experimental system is in use at Washington University to screen for compounds that modulate $A \beta$ levels in vivo. Even though the in vivo microdialysis technology in the study has not been patented, Cirrito said his team is "definitely open to collaborating with industry."

\section{Second BACE}

What happens to APP after endocytosis was the subject of the second study, published in Science by a team of industry and academic researchers in Germany. They showed that they could greatly improve the specific activity of an inhibitor of a key APP processing enzyme$\beta$-secretase (BACE1) - by slipping it into endosomes.

BACE1 is a protease that is active inside of endosomes. Along with $\gamma$-secretase, BACE1 digests endosome-bound APP into the form that eventually is secreted as $A \beta$.

Though pharma companies including GlaxoSmithKline plc, Merck \& Co. Inc. and Wyeth have been in the BACE space for years, not one compound has reached the clinic. And until now, no company has specifically targeted endosomes with BACE1 inhibitors.

The Science study presented an improved BACE1 inhibitor that concentrates in specialized membrane structures called lipid rafts. Lipid rafts are naturally occurring clusters of unusual lipids: they harbor many membrane proteins destined for endocytosis, including APP. ${ }^{6}$

The study was led by Kai Simons, professor and executive director of the Max Planck Institute of Molecular Cell Biology and Genetics 
Figure 1. Mapping early Alzheimer's disease events and targets. Recent studies elucidating the sequence of steps related to the production of $\beta$-amyloid $(A \beta)$ and its effect on downstream signaling pathways highlight new therapeutic targets, including $\beta$-secretase (BACE1), $N$-methyl-D-aspartic acid-type glutamate receptors (NMDARs) and synaptojanin 1 (Synj1). In the sequence, amyloid precursor protein (APP) associates with lipid rafts in the synaptic membrane [a]. Upon neuronal activation, raft-bound APP is internalized [b] into endosomes. BACE1, together with $\gamma$-secretase, cleaves APP [c] to form the truncated fragment $A \beta_{42}$ that returns to the synaptic membrane [d] and is secreted. $A \beta_{42}$ then assembles into small oligomers [e] that activate NMDARs [f]. This leads to activation of phospholipase C (PLC) [g], which converts the membrane-bound phospholipid phosphatidylinositol-4,5-bisphosphate $\left(\mathrm{PIP}_{2}\right)$ into inositol triphosphate $\left(\mathrm{IP}_{3)}[\mathrm{h}]\right.$. In turn, $\mathrm{IP}_{3}$ activates further signaling pathways that lead to neuron death. The lipid phosphatase Synj1 also lowers $\mathrm{PIP}_{2}$ levels, promoting toxicity [i].

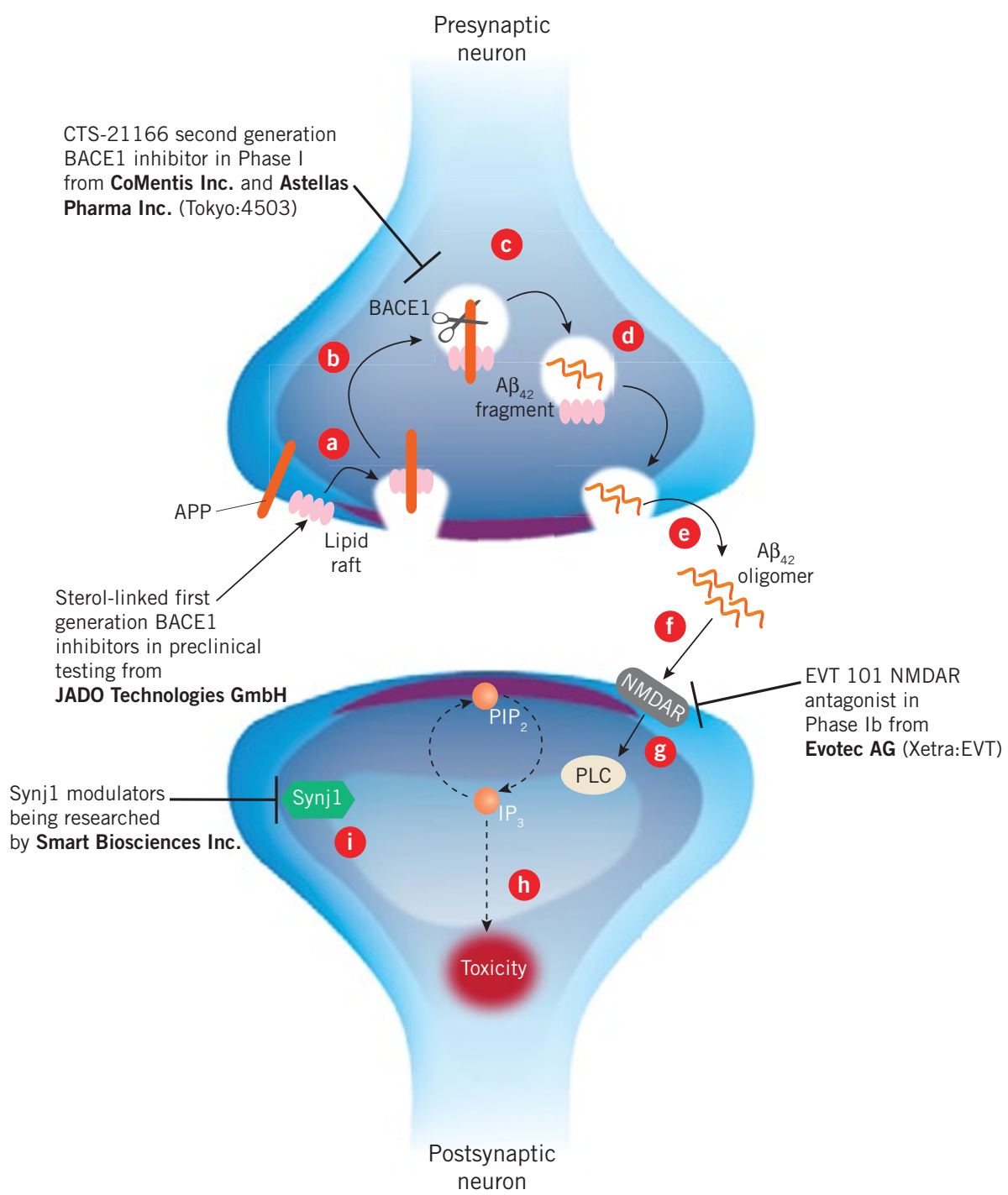

and a founder of JADO Technologies $\mathbf{G m b H}$, which is developing technology for targeting therapeutics to lipid rafts. ${ }^{7}$ Jado scientists coauthored the Science paper.

The company's lead lipid raft-targeting compound, TF002, is in Phase II trials to treat cutaneous mastocytosis and atopic dermatitis.

Simons' team used cultured cells and fly and mouse models to create a bioavailable BACE1 inhibitor. Simons told SciBX that despite success with in vitro BACE1 inhibitors, companies are not delivering their compounds to the right place in the cell.

"We know that BACE1 cleaves APP after endocytosis, so a BACE1 inhibitor has to get into the endosomes," he said.

The team found that using a fatty acid lipid as an anchor for its BACE1 inhibitor did not work. Only a sterol anchor, which sought out lipid rafts, proved effective at inhibiting BACE1 in vitro and in vivo.

The anchored BACE1 inhibitor was preferentially taken up into endosomes, where it blocked APP cleavage and boosted the survival of APP-transfected cultured cells compared with the effects of soluble BACE1 inhibitor.
Also, the sterol-BACE1 formulation improved survival in a fly AD model compared with survival of mock-treated flies. When the formulation was injected into the brains of APP transgenic mice, the compound lowered hippocampal $A \beta$ levels compared with those in mock-treated mice.

The lipid raft-targeting technology in the study is patented by Max Planck and by the Technical University of Dresden and is licensed to Jado.

The Science report underscores the importance of lipid rafts in disease and validates Jado's therapeutic approach, according to CEO Charl van Zyl. He said lipid rafts have not previously been considered good drug targets because of their complex composition and dynamic nature.

"We are a bit of a lone voice," said Zyl.

Although Jado is the only company going after lipid rafts, the company is not the only one pursuing BACE1. An alternative approach, taken by CoMentis Inc., involves optimizing the chemistry and bioavailability of soluble BACE1 inhibitors to achieve the same goal of BACE1 inhibition without using a lipid anchor. 
Martin Tolar, CSO of CoMentis, called the Simons paper a new "proof-of-concept-type study in the absence of other BACE1 inhibitors." Though the compound is promising, he cautioned it will require "a lot of basic pharmacology and optimization" of brain penetration, oral bioavailability and other parameters.

Earlier this month, CoMentis granted Astellas Pharma Inc. exclusive worldwide rights to develop and commercialize products from CoMentis' BACE1 inhibitor program, including CTS-21166, which has completed a Phase I trial to treat $\mathrm{AD}$. CoMentis will receive $\$ 80$ million up front plus a $\$ 20$ million equity investment and is eligible for $\$ 660$ million in development milestones, plus commercial milestones and royalties.

Simons and Zyl agreed that their preclinical findings are just the beginning. Their formulation has yet to be given a name and must be tested in mice to determine if it can penetrate the blood-brain barrier before Phase I trials can begin.

\section{$\mathrm{PIP}_{2}$ squeak}

The third study, published in Nature Neuroscience, uncovered the effects of $\mathrm{A} \beta$ after it leaves the endocytic pathway. The paper identified a regulatory phospholipid that is disrupted by $\mathrm{A} \beta$ exposure, as well as other potential therapeutic targets to prevent this toxic effect.

In the synapse and other extracellular spaces, $A \beta$ assembles into small oligomeric aggregates that are thought to be precursors of the larger amyloid fibrils found in AD plaques. Oligomers are highly toxic to neurons, but how they kill is poorly understood.

The Nature Neuroscience study was performed by a team led by Tae-Wan Kim, assistant professor of pathology at the Taub Institute for Research on Alzheimer's Disease and the Aging Brain at Columbia University Medical Center, and by Di Paolo.

Kim and Di Paolo exposed primary cultured mouse neurons to synthetic $A \beta$ oligomers and monitored the levels of various phospholipids in cell membranes. The researchers found that $A \beta$ exposure rapidly reduced the level of $\mathrm{PIP}_{2}$ compared with that in untreated controls.

$\mathrm{PIP}_{2}$ is a signaling molecule associated with the plasma membrane. ${ }^{8}$ When upstream pathway components are activated, $\mathrm{PIP}_{2}$ is converted into inositol triphosphate $\left(\mathrm{IP}_{3}\right)$, a soluble lipid that travels from the membrane to the cytoplasm. There, $\mathrm{IP}_{3}$ stimulates the release of calcium into the cell from the endoplasmic reticulum. High intracellular calcium has previously been implicated in AD. ${ }^{9}$

The likely explanation for why $\mathrm{A} \beta$ lowered $\mathrm{PIP}_{2}$ levels is because $\mathrm{PIP}_{2}$ was being converted into $\mathrm{IP}_{3}$.

The researchers tested this hypothesis with a small molecule inhibitor of phospholipase C (PLC), a common signaling enzyme that converts $\mathrm{PIP}_{2}$ to $\mathrm{IP}_{3}$, as well as with a selective inhibitor of the $N$-methyl-D-aspartic acid receptor (NMDAR). The NMDAR is a membrane-bound sensor that activates PLC in response to the neurotransmitter glutamate.

In both cases, the researchers found that $A \beta$ no longer lowered $\mathrm{PIP}_{2}$ levels.
Encouraged by these findings, Kim and Di Paolo chose to focus on a more downstream target-a lipid phosphatase called synaptojanin 1 (Synj1) - in hopes that Synj1's effects would not be as widespread as those of PLC. Synj1 antagonizes PIP ${ }_{2}$ levels and thus inhibiting Synj1 should lead to more PIP ${ }_{2}$.

Indeed, the Columbia team found that mice with a single functional copy of Synj1 did not show lowered PIP ${ }_{2}$ levels upon A $\beta$ treatment and were resistant to some of the toxic effects of $A \beta$.

Boosting $\mathrm{PIP}_{2}$ levels thus may be a new therapeutic strategy for treating $\mathrm{AD}$, according to the authors. "We believe that partial inhibition of the enzymes involved in the downregulation of $\mathrm{PIP}_{2}$ could prevent some of the effects of A $\beta$," Di Paolo told SciBX.

However, the researchers told SciBX that PLC itself is likely too important an enzyme in normal biology to be a good drug target.

The NMDAR is already being targeted by Evotec AG's EVT 101. In April, the company reported that the compound was well tolerated in a Phase Ib trial with 19 healthy volunteers. It produced no improvements in cognitive function, which Evotec said was expected because healthy young subjects "frequently perform at an optimum level." However, it did produce changes in local cerebral blood flow to brain regions that the company said are involved in memory retrieval.

Kim and Di Paolo are choosing to target Synj1 with the help of Smart Biosciences, which Kim founded. The company, which has $\$ 2$ million in series A funding, plans to develop chemical modulators of Synj1 and screen for compounds that affect $\mathrm{PIP}_{2}$ levels by hitting other targets.

Smart's lead compound is SMT-3, a small molecule modulator of phosphoinositide synthesis. According to Kim, SMT-3 is still at the "proof-of-concept stage."

Kim and Di Paolo's discoveries are patented by Columbia University and licensed to Smart Biosciences. The company is seeking to outsource future medicinal chemistry to industry collaborators in Korea.

Korea has "good IP protection, an emerging pharmaceutical market and a very good clinical trial infrastructure," said Kim. Korea is "cost-effective for collaboration."

\section{Bringing it all home}

The prospects for targeting $\mathrm{PIP}_{2}$ remain unclear until key next experiments are done. The biggest question is whether Synj1 loss of function prevents $\mathrm{A} \beta$ toxicity by blocking $\mathrm{IP}_{3}$ production and the release of intracellular calcium or by some other mechanism.

David Hung, CEO of Medivation Inc., agreed that the Kim and Di Paolo study shows that $A \beta$ "oligomers are pretty potent inhibitors of $\mathrm{PIP}_{2}$ signaling."

However, Hung suggested the phosphoinositide pathway may be unsuitable for drug targeting because of its importance. "Many cells use that pathway, and inhibiting it will have a multitude of significant consequences," he said.

Hung said Medivation's Dimebon compound may be a better choice for treating the cellular mechanism of AD. In AD, excessively 
high intracellular calcium enters the mitochondria, activating apoptotic signals that lead to cell death. Dimebon prevents this process by blocking the mitochondrial permeability transition pore.

"We don't block the calcium inside of the cell, we just block mitochondrial entry" of the calcium, said Hung.

In March, Dimebon met the primary and secondary endpoints of Alzheimer's disease assessment scale-cognitive subscale and clinician interviews, respectively, in a Russian Phase II trial. Medivation expects to launch a Phase III trial this year.

In addition, Washington University's Cirrito pointed out that the Kim and Di Paolo study did not show that modulating PIP ${ }_{2}$ levels could protect against $A \beta$ endogenously produced by neurons. At least some of the findings could relate to the synthetic origin of the $\mathrm{A} \beta$ oligomers used in their study or to the way in which the oligomers were administered.

"Making $A \beta$ synthetically or in culture isn't exactly what you find in a brain," said Cirrito.

Another concern is that Synj1 has been previously implicated in endocytosis at the presynaptic membrane. Thus, Synj1 mutants could potentially have lower $A \beta$ levels because of a defect in endocytosis rather than through differences in $\mathrm{PIP}_{2}$ levels. ${ }^{10}$

Di Paolo thinks that the specific location of Synj1 activity may not matter because both sides of the synapse can make and take up A $\beta$ oligomers.

"The machinery of pre- and postsynaptic membranes is largely the same," he said.

Di Paolo agreed that the key next experiment to address these questions is to cross the Synj1 mutant mice with an $A \beta$ transgenic strain. This should establish whether modifying PIP 2 metabolism translates into histological and cognitive improvement in real life.

\section{REFERENCES}

1. Cirrito, J.R. et al. Neuron; published online April 9, 2008; doi:10.1016/j.neuron.2008.02.003

Contact: John R. Cirrito, Washington University School of Medicine, St. Louis, Mo.

e-mail: cirritoj@neuro.wustl.edu

2. Rajendran, L. et al. Science; published online April 25, 2008; doi:10.1126/science.1156609

Contact: Kai Simons, Max Planck Institute of Molecular Cell Biology and Genetics, Dresden, Germany e-mail: simons@mpi-cbg.de

3. Osherovich, L. SciBX 1(4), 1-4; Feb. 21, 2008

4. Berman, D.E. et al. Nat. Neurosci.; published online April 6, 2008; doi: $10.1038 / \mathrm{nn} .2100$

Contact: Tae-Wan Kim, Columbia University Medical Center, New York, N.Y.

e-mail: kwk16@columbia.edu

Contact: Gilbert Di Paolo, same affiliation as above e-mail:gil.dipaolo@columbia.edu

5. Liu, Q. et al. Neuron 56, 66-78 (2007)

6. Allen, J.A. et al. Nat. Rev. Neurosci. 8, 128-140 (2007)

7. Maggos, C. BioCentury 15(20), A15; April 30, 2007

8. Di Paolo, G. \& De Camilli, P. Nature 443, 651-657 (2006)

9. Thibault, O. et al. Aging Cell 6, 307-317 (2007)

10. LaFerla, F.M. et al. Nat. Rev. Neurosci. 8, 499-509 (2007)

COMPANIES AND INSTITUTIONS MENTIONED

Astellas Pharma Inc. (Tokyo:4503), Tokyo, Japan

Evotec AG (Xetra:EVT), Hamburg, Germany

Columbia University Medical Center, New York, N.Y.

CoMentis Inc., South San Francisco, Calif.

GlaxoSmithKline plc (LSE:GSK; NYSE:GSK), London, U.K.

Jado Technologies GmbH, Dresden, Germany

Max Planck Institute for Molecular Cell Biology and Genetics,

Dresden, Germany

Medivation Inc. (NASDAQ:MDVN), San Francisco, Calif.

Merck \& Co. Inc. (NYSE:MRK), Whitehouse Station, N.J.

Smart Biosciences Inc., Philadelphia, $\mathrm{Pa}$.

Technical University of Dresden, Dresden, Germany

Washington University School of Medicine, St. Louis, Mo.

Wyeth (NYSE:WYE), Madison, N.J. 